\title{
EEG Spike Detection using Stationary Wavelet Transform and Time-Varying Autoregressive Model
}

\author{
Mehdi Radmehr \\ Islamic Azad University, Sari Branch, Iran
}

\author{
Seyed Mahmoud Anisheh \\ Faculty of Electrical Engineering of \\ K. N. Toosi University of Technology, Tehran, Iran
}

\begin{abstract}
Spikes are short-time broadband events, which can last $20 \mathrm{~ms}-$ $70 \mathrm{~ms}$ and amplitude is among $100 \mu \mathrm{V}-200 \mu \mathrm{V}$. In this research, a novel spike detection method based on stationary wavelet transform (SWT) and time-varying autoregressive model is proposed. In the proposed method, the discrete stationary wavelet transform is initially applied on the signal under analysis to show the important underlying unadulterated form of the data. The time-varying AR (TVAR) is used as an effective tool for analyzing non-stationary signals such as spikes. The performance of the proposed method is compared with other existing methods using both synthetic signals and real newborn Electroencephalogram (EEG). The simulation results indicate the absolute advantages of the proposed method.
\end{abstract}

Keywords: Spike detection, Stationary wavelet transform, Time-varying autoregressive model, EEG.

\section{INTRODUCTION}

Spikes in EEG are usually an indication of abnormal functioning of brain activity [1-3]. Brain functioning affects the morphology of EEG. Spikes can be defined as transient signals, clearly distinguishable from the background activity by a pointed peak with a duration varies between 20 and 70 ms [1-3]. Seizures, which are a result of synchronous discharge of a large number of neurons, can be detected using spikes and their firing pattern in EEG [1-3]. From the signal processing point of view, the detection of spikes is an important problem in many biomedical Applications.

Due to signal to noise ratio, the presence of noise may prevent recognition and localization of spikes. There are several spike detection methods in the literature. In [4], a spike detection method based on relative amplitude of the waveforms has been suggested. In this approach, only waves whose amplitudes are at least six times bigger than that of the background are considered as spike signals. However, it fails to detect small amplitude spikes after burst waves. In [5], the authors proposed a spike detection method using a nonlinear energy operator (NEO). The NEO can be used for amplifying the spiky activities in a background signal. However, the NEO is sensitive to noise and has the problem of cross terms [6]. In another method, namely SNEO, the output of the NEO is convolved with a Barlett window in order to eliminate the spurious spikes due to the cross terms and background noise [6]. In [7], the EEG signal is initially is modeled using a Kalman filter. Then, the output values of the filter above the threshold are considered as the positions of spikes. However, this method has more false detection rate. In this research, a new spike detection technique is presented. Discrete stationary wavelet transform (SWT) is used as a preprocessing stage to enhance the accuracy of the proposed method. The time-varying AR (TVAR) model can be employed for transient detection in non-stationary signals as well as stationary ones.
This article is organized as follows: Section 2 clarifies the proposed method. The performance evaluation of the proposed method and comparison results are provided in Section 3. Finally, the conclusions are given in Section 4.

\section{PROPOSED SPIKE DETECTION METHOD}

I. The discrete wavelet transform analyses the signal at different frequency bands with different resolutions by decomposing the signal into the so-called approximations and details. The decomposition of the signal into different frequency bands is simply obtained by low-pass and high-pass filtering of the time domain signal $[8,9]$. In this approach, the obtained results at each level are half the size of the original sequence. The SWT, on the other hand, pads the corresponding low-pass and high-pass filters with zeros to retain the length of the signal. Consequently, the major advantage of SWT is the preservation of time information of the original signal sequence at each level. This property is useful for several applications such as breakdown point's detection.

II. In this method two sliding windows move alongside the entire signal. The signal in each window of this method is modeled by a TVAR model instead of the conventional AR which is only applicable for stationary signals. In the standard AR structure, a discretely sampled signal is modeled by representing the voltage level at time $\mathrm{t}$ as a linear combination of voltage levels at times $\mathrm{t}-1, \mathrm{t}-2, \ldots, \mathrm{t}-\mathrm{p}$ for $\mathrm{p}>0$ an addition a random (driving noise) component. The relationship is supposed to be fixed over time in that the regression parameters defining the linear combination are constant for the whole period of recording. While in the TVAR model these parameters differ over time, adapting to changes evidenced in the signals, and therefore, potentially provides the kinds of time evolving structure evident in many nonstationary signals. Such models can specially answer to and adequately capture the forms of change in the frequency structure of oscillations in EEG data [10]. If the sliding windows fall within a location of background signal, since the both windows have the same statistical properties, the modeling error between the two windows is low. However, if both sliding windows are placed in the position of spikes, the modeling error rises.

III. Determining a threshold is the final step in finding the position of spikes. In many researches, the mean value or the mean value added to standard deviation or something like those are proposed as a threshold. If the defined threshold is large, several positions of spikes may not be indicated. While the threshold value is low, several positions of spikes may be selected inaccurately. In this paper the threshold value is chosen as $\bar{V}+\sigma_{V}$, where $\bar{V}$ and $\sigma_{V}$ represent mean and standard deviation of the output of TVAR function, respectively. When the local maximum is bigger than the 
threshold, the current time is selected as an indication of spike.

\section{PERFORMANCE EVALUATION}

Algorithm of the proposed method and existing techniques were implemented using MATLAB from Math Works, Inc. The performance of these techniques was evaluated using both synthetic signal and real newborn EEG.

\subsection{Synthetic Signal}

For the purpose of evaluating the performance of the proposed method, the following synthetic signal has been generated [11]:

$$
x(t)=h(t)+s(t)
$$

where $h(t)$ and $s(t)$ are the spike train set and the background signal, respectively. The background signal is chosen according to equation (2).

$$
s(t)=\sin (\omega t)-\sin (2 \omega t+\phi)+\sin (4 \omega t)+n(t)
$$

where $\omega=2 \pi / 75, \phi=\pi / 2$ and $n(t)$ is white Gaussian noise. The spikes are distributed randomly over the background signal. Spike signals are modeled as triangular symmetric pulses whose amplitudes were uniformly distributed in the interval of 2.5 and 5. The signal is sampled at a rate of $128 \mathrm{~Hz}(\mathrm{Fs}=128 \mathrm{~Hz})$. The signal in Figure 1.a represents 8 spikes with a randomly varying duration of 3 to 9 samples since the duration of real spikes ranges from 20 to 70 ms. Figure 1.b shows 2 seconds of $x(t)$ with $S N R=0 d B$.

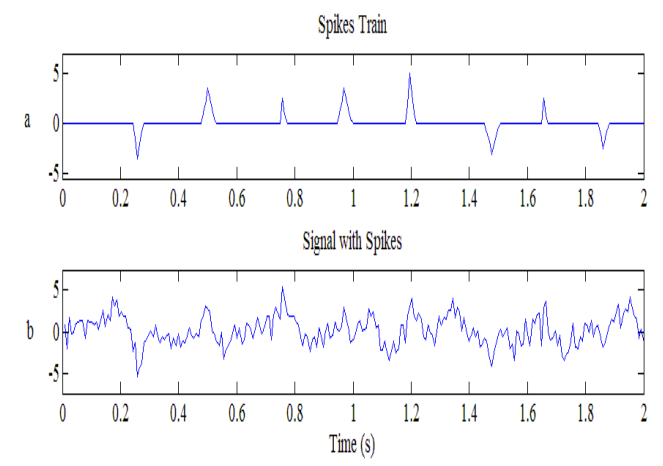

Figure 1. (a) Spikes train. (b) Signal with spikes, $x(t)$ with SNR=0 dB.

We have decomposed the signal in Figure 2.a using a onelevel SWT, and the approximate sub-band is used in the next step. In this research, SWT is performed with Daubechies wavelet of order 8 . The TVAR output has been computed and then predefined threshold has been applied on it. The threshold value for the proposed method is chosen as $\bar{V}+\sigma_{V}$, where $\bar{V}$ and $\sigma_{V}$ represent mean and standard deviation of the output of TVAR function, respectively. As seen from the Figure 2, the proposed method clearly represents the positions of 6 spikes. The position of spikes has been depicted by arrows in Figure 2.c.

In order to evaluate the performance of the proposed method, miss or false negative (FN) and false alarm or false positive (FP) ratios are used as defined below [11]:

$$
F N=\frac{N_{m}}{N}, \quad F P=\frac{N_{f}}{N},
$$

where $\mathrm{Nm}, \mathrm{Nf}$ and $\mathrm{N}$ represent the number of missed, falsely detected and actual number of spikes, respectively.

Table 1 reports the FN and FP ratios obtained by applying the proposed method and the existing methods to 100 simulated signals with $10 \mathrm{~dB}, 100$ simulated signals with $5 \mathrm{~dB}, 100$ simulated signals with $0 \mathrm{~dB}$ SNR. As can be seen from the results, the proposed method has a better accuracy compared to the existing methods $[6,7]$. The proposed method approach has the lowest value for FN ratio in each selected SNR. In addition, it has lowest value for FP ratio especially for the lower SNR.
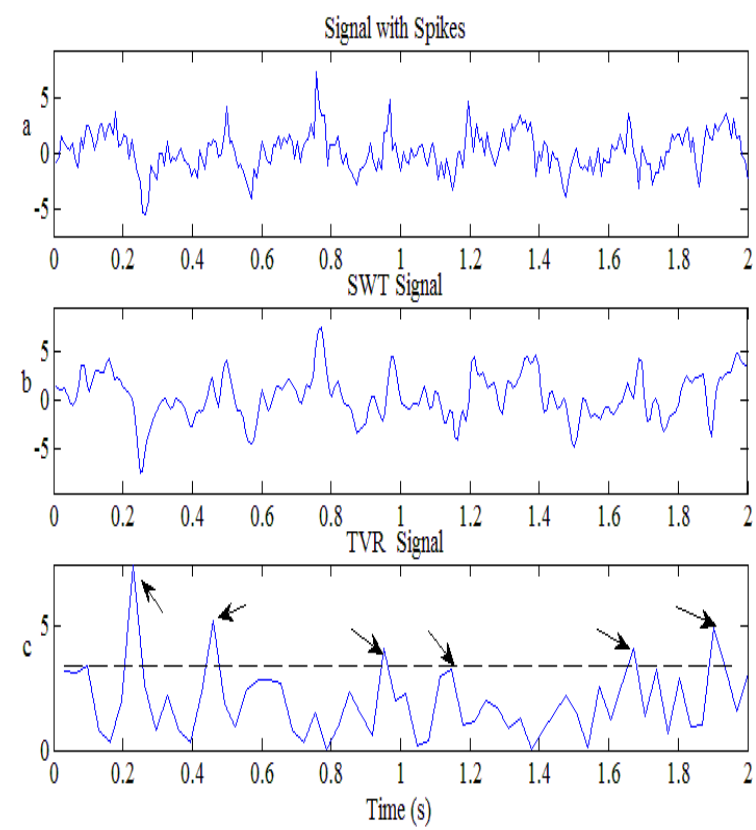

Figure 2. Spike detection in synthetic signal using proposed method. (a) Signal with spikes. (b) Approximate sub-band of SWT signal, (c) Output of the TVAR signal. As can be seen, the proposed method could successfully detect 6 spikes.

Table 1. Results of applying proposed methods and the existing methods on a set of synthetic data.

\begin{tabular}{|c|c|c|c|}
\hline \multicolumn{4}{|c|}{ Proposed Method } \\
\hline SNR & $0 \mathrm{~dB}$ & $5 \mathrm{~dB}$ & $10 \mathrm{~dB}$ \\
\hline FN & 0.25 & 0.11 & 0.02 \\
\hline FP & 0.13 & 0.08 & 0.04 \\
\hline \multicolumn{4}{|c|}{ SNEO Method } \\
\hline SNR & $0 \mathrm{~dB}$ & $5 \mathrm{~dB}$ & $10 \mathrm{~dB}$ \\
\hline FN & 0.36 & 0.17 & 0.08 \\
\hline FP & 0.41 & 0.21 & 0.11 \\
\hline \multicolumn{5}{|c|}{ Kalman Based Method } \\
\hline SNR & $0 \mathrm{~dB}$ & $5 \mathrm{~dB}$ & $10 \mathrm{~dB}$ \\
\hline FN & 0.46 & 0.17 & 0.08 \\
\hline FP & 0.59 & 0.34 & 0.25 \\
\hline
\end{tabular}




\subsection{Real EEG Signal}

For the purpose of evaluating the performance of the proposed method on real signal, the EEG of a newborn baby has been used. The signal have been sampled using Fs $=256 \mathrm{~Hz}$. The proposed method is applied to five seconds of EEG signal and the result is shown in Figure 3. The position of the spikes has been depicted by arrows in Figure 3.a. The output of SWT signal and the calculated TVAR signal are shown in Figure 3.b and Figure 3.c, respectively. It is clear that that all the spikes have been successfully detected using the proposed method.

\section{CONCLUSIONS}

In this paper a new spike detection methods has been introduced. Wavelet transform reveals the important underlying unadulterated form of the signal and TVAR is a powerful approach in transient detection of non-stationary signal. The performance of the proposed method was compared with other exiting methods using both synthetic signals and data collected from human subjects. Simulation results indicated proposed methods outperforms the existing methods in spike detection.
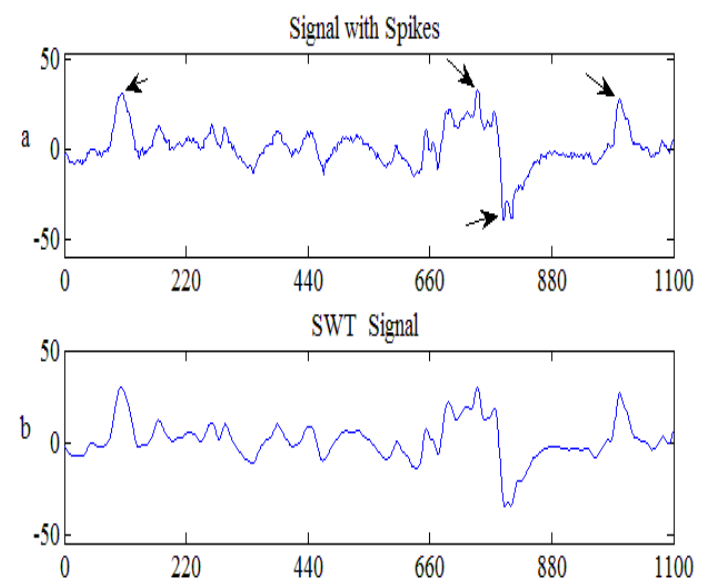

TVAR Signal

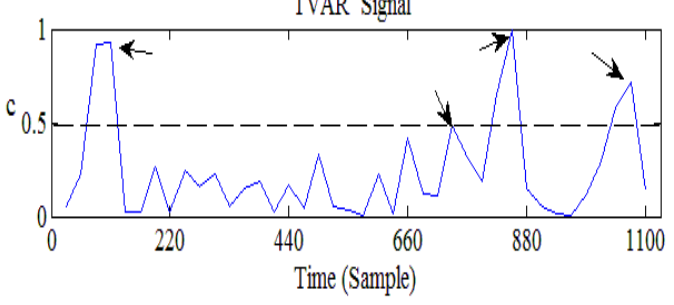

Figure 3. Spike detection in real signal using proposed method. (a) Signal with spikes. (b) Approximate sub-band of SWT signal, (c) Output of the TVAR signal. As can be Seen, the proposed method could successfully detect all spikes.

\section{REFERENCES}

[1] Hassanpour, H., Mesbah, M., and Boashash, B. 2004. "Time-frequency based newborn EEG seizure detection using low and high frequency signatures," Physiological Measurement. vol. 25, pp. 935-944.

[2] Hassanpour, H., Mesbah, M., and Boashash, B. 2003. "Comparative performance of time-frequency based newborn EEG seizure detection using spike signatures," Proc. IEEE Int Conference on Signal Processing, vol.2, pp. 389-392.

[3] Xu, G., Wang, J., Zhang, Q., Zhang, S., and Zhu, J. 2007. "A spike detection method in EEG based on improved morphological filter," Computers in Biology and Medicine, vol. 37, pp. 1647-1652.

[4] Wilson, S.B., and Emerson, R. 2002. "Spike detection: a review and comparison of algorithms," Clinical Neurophysiology, vol. 113, pp. 1873-1881.

[5] Maragos, P., Kaiser, J.F., and Quatieri, T.F. 1993. "On amplitude and frequency demodulation using energy operators", IEEE Trans. Signal Processing, vol. 41, pp. $1532-1550$

[6] Mukhopadhyay, S., and Ray, G.C. 1998. "A new interpretation of nonlinear energy operator and its efficiency in spike detection," IEEE Trans. On Biomed.Eng., vol.45, pp.180-187.

[7] Tzallas, A.T., Oikonomou, V.P., and Fotiadis, D.I. 2006. "Epileptic Spike Detection Using a Kalman Filter Based Approach," Proc. IEEE Int EMBS Conference, vol.1, pp. 501-504.

[8] Dimoulas, C., Kalliris, G., Papanikolaou, G., and Kalampakas, A. (2007), 'Long-term Signal Detection, Segmentation and Summarization Using Wavelets and Fractal Dimension: A Bioacoustics Application in Gastrointestinal-Motility Monitoring', Computers in Biology and Medicine, 37, 438-462.

[9] Yamaguchi, C. (2003), 'Fourier and Wavelet Analyses of Normal and Epileptic Electroencephalogram (EEG)', In Proceedings of the 1st International IEEE EMBS.

[10] Krystal, A. D., Prado, R., and West, M. 1999. "New methods of time series analysis of non-stationary EEG data: eigenstructure decompositions of time varying autoregressions" Clinical neurophysiology, vol. 110 no. 12, pp. 2197-206.

[11] Malarvili, M., Hassanpour, H., Mesbah, M., and Boashash, B. 2005. "A histogram-based electroencephalogram spike detection," Proc. IEEE Int Symposium on Signal Processing and its App, vol.1, pp. 207-210. 